\title{
Research on Key Issues in Future Wireless Network
}

\author{
Chih-Lin $\mathrm{I}^{1}$, Yucheng Zhang ${ }^{1,2}$, Jinglin Shi $^{1}$, Mugen Peng ${ }^{3}$, Branka Vucetic ${ }^{4}$ \\ ${ }^{1}$ (Institute of Computing Technology, Chinese Academy of Sciences, Beijing, China, 100190) \\ ${ }^{2}$ (Graduate University of Chinese Academy of Sciences, Beijing, China, 100049) \\ Email: \{icl, Yucheng Zhang, sjl\}@ict.ac.cn \\ ${ }^{3}$ (Wireless Signal Precessing and Network Laboratory Beijing University of Posts and telecommunicationsBeijingChina ) \\ Email:pmg@bupt.edu.cn \\ ${ }^{4}$ (School of Electrical and Information Engineering University of Sydney, Australia) \\ Email:branka@ee.usyd.edu.au
}

\begin{abstract}
Based on the investigation of the phenomenon in complex networks, this paper proposed some important facing problems in future wireless networks, that is, it is difficult to fully predict the trend of wireless networks from the perspective of application requirements and the technology development. Therefore, with the hot spots in future wireless network, a research framework was proposed which was made from three levels including philosophy, science of system and technologies. In the study of this framework, three levels of contents are proposed, that is, service models research which related to social science, the network control model research which related to system science, and the transmission model research which related to nature science. Some key technologies are deplored based on the methodology proposed in this paper.
\end{abstract}

\section{INTRODUCTION}

In the past few decades, wireless network research has become one of the hot spot in engineering technologies. With the appearance of increasingly large-scale wireless networks, people are feeling more and more difficult to explain complex phenomenon due to lack of effective methods. However, complex phenomena occurring in some technology or social networks provides a new approach to research into wireless network. For example, massive power blackout caused by minor disturbances in North America in the year of 2003 continued for tens of hours, which turned the whole United States and Canada into panic[1]. If target attacks occurred during this period, the loss would be immeasurable[2,3]. Similar power outage occurred in several countries every years and caused the attention of researchers. Another example is virus propagation in both social network and computer network. As people migration become frequent in modern society, the model and speed of virus propagation has shown some common features [4][5][6][7]. The third class of complex phenomenon is the network emerge in special network structure, which undermines the infrastructure of Internet and society. In view of such threats, more and more governments are beginning to pay attention to network monitoring[8][9].

Although these phenomena occurred in different technical networks or social networks, they have common features as follows:

a) All of the phenomena occurred in huge complex network systems. b) Minor disturbance may have enormous impact on huge network.

c) Problems can not be easily solved from pure technical perspective, which ask for new methods from higher levels.

Similar phenomenon also appears in many other kinds of networks such as transport network, biological network, dam network and so on. Due to habitual thinking, researchers are accustomed to study these problems from technical perspective, and attempt to optimize the design of network infrastructure to bring the network into stable status. However, technology methods are hardly competent to explain phenomenon in large-scale systems such as chaos, fractal phenomena and so on. Thereafter, traditional technology methods combined with social views provide a new direction for future wireless network research, which makes the successful design of optimum wireless network and serving future society become possible.

This article investigates into the development history of wireless network technology, and discussed possible methods and content of future wireless network research. It is organized as follows. In section II, the development processes of network system, specifically wireless network are reviewed. In section III, the research methods and contents are discussed from the perspective of philosophy. The system-level research contents and methods are discussed in the section I V and the technicallevel research in the section V. Finally, summarize the full text.

\section{Wireless Network Research Methodology EXPLORE}

The development of future wireless network can be driven by technology innovation or application requirement. As shown in figure 1, network technologies have evolved over half a century with the initial appearance of narrow band network, until current broad band network and future universal intelligent system. Transmission technology have developed from frame-relay, to ethernet and fibre network, while network technologies have developed from IPv4, IPv6 and post-IP, and application requirement are from email, audio, video and future universal application data. As the characteristics of future wireless network cannot be predicted from any kind of existing technology, the prediction of wireless network from technical point has been questioned for a long time. 


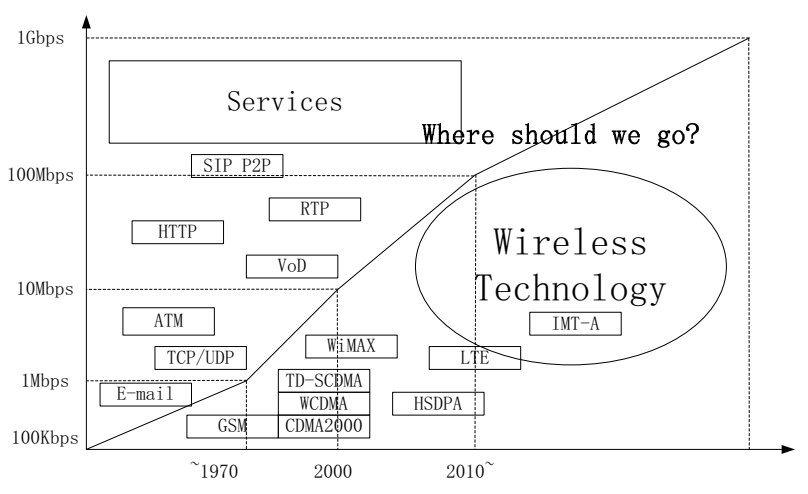

Fig. 1. Technology Forecasting

Therefore, new tools used for future wireless network research should be taken into account besides application demands and technologies development. Firstly, philosophy methodology should be explored to investigated into the external environment of network system. Issues on future network morphology should combine system behavior and network structure. Lastly, based on above work, supporting technologies for future wireless network can be predicted. Eventually, a research system for future wireless network can be built up from philosophy, network dynamics and technologies.

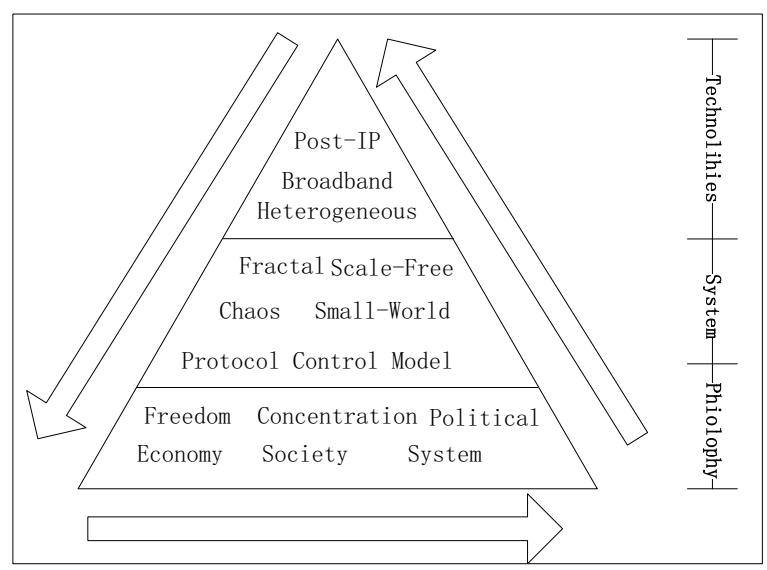

Fig. 2. The research system of Future wireless network

As shown above, from the system view, firstly,we need to recognize the external environment to better predict future wireless network development. The external environment can affect the overall behavior of system. The research of external environment of the network system needs to integrate with social, economic and cultural trends, in order to inspect into system growth. Any technologies will reflect social attributes to some extent. For example, BGP protocol is based on U.S. election system as a prototype. Internet itself reflects the western values of freedom. Thus, in order to better study the future wireless network, the first step is to study the society from philosophical point of view. So the purpose of the network environment research is to investigate into the philosophy of future wireless network construction.

As shown in figure 2, studies of network dynamics include network structure and behavior models [12]. After several decades of development, Internet has grown into a huge complex system. Many complex phenomena have shown such as worm propagation, network traffic fractal, chaos, small world characteristic of network structure. With the appearance of thing-Internet, intelligent control and multi-scale transmission, future wireless network has to cope with more and more system behavior problem. Therefore, it is necessary to study network infrastructure from network dynamics view. These research contents have now been carried out as an independent exploration and research subjects.

The third level of network research is technologies research. Under the guidance of new research ideas [13], the internet of thing, triple play and post IP will be the main driving force of technological development. The new round of eless network technologies revolution will be formed, details includes innovation service platform, network control model and multi-scale transport model.

\section{STUDY ON THE PHILOSOPHY OF FUTURE WIRELESS NETWORK}

From the perspective of philosophy, the main contents of wireless network are the research on system framework and its social attribute analysis with consideration of the environment of network technologies. In addition to its natural attributes, network system and technologies contain some hidden social attributes. So the main work of studying network system in a philosophical way is to excavate and analyze its social attributes, then establish the guiding ideology of its framework. Nowadays, the concepts of communication network framework can be divided into three categories. The first is to strengthen network control and management, construct a close and stable system by combing the transmission, control and management together. For example, the mobile telecommunication network is a highly stable system constructed under this principle, and also ITU use this guiding principle to lead the innovative communication technologies worldwide. The second way to construct a communication network is using the freedom and "do-nothing" concept. The Internet is a typical example under this concept. Based on the open but unified system, it provides a verification platform for any innovative technology and idea worldwide. Undeniably, this "do-nothing" concept provides a wide space for the development of network technologies and its outspread evolution. But the disadvantage of this open platform is lack of stability of management.

For the next generation network, we have to explore a third way by using "the doctrine of the mean", for any extreme guiding ideology will leave hidden dangers to the network. At present, we abandon the "on the edge" and "in the center" arguments, and divide the network capacity according to the development trend of electronic technology. In the close and open network framework, we intensify the control of core 
functional nodes and throw open the parts which is close to the users. Then the network can be easily and flexibly controlled.

The greatest advantage of the robust design concept of future wireless network is to keep its distinctive features while leaves much room for a flexible framework at the same time. So in the network framework design, we have to provide a revolutionary upgrading concept by using philosophical methods which can avoid the network development from a blind alley.

\section{DyNAMics AND STRUCTURE OF FUTURE WIRELESS NETWORK RESEARCH}

The study object of future wireless network dynamics is the special behavior of network system, such as self-similar traffic, architecture complexity, spread characters of virus, et al. Study of future wireless network needs theories of statistical physics, fluid physics and system science. Currently, hotspots and difficult points in this field are as follows: Evolution and change law of wireless network architecture; Linkage model of special network system architecture and data traffic; characters of energy distribution and flow behavior of wireless network in multidimensional space and the control method of network structure improvement to virus spreading. All these about architecture evolution of future wireless network.

Study methods mainly rely on computer simulation. First, build a wireless network simulate platfrom on which to simulate service flows, especially about generating and shifting model of massive data. Furthermore, simulate the integration and impact effect to network structure of data traffic for studying of the impact of network structure evolution on the traffic carrying capacity, and then optimize the network system architecture.

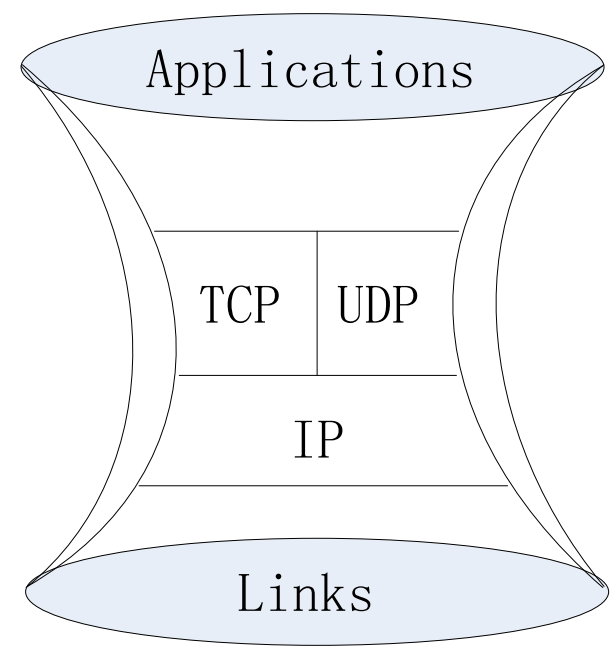

Fig. 3. Hourglass Protocol Mode

Based on the research achievements of dynamics, the future wireless network architecture study mainly includes two aspects, protocol model framework and wireless networking

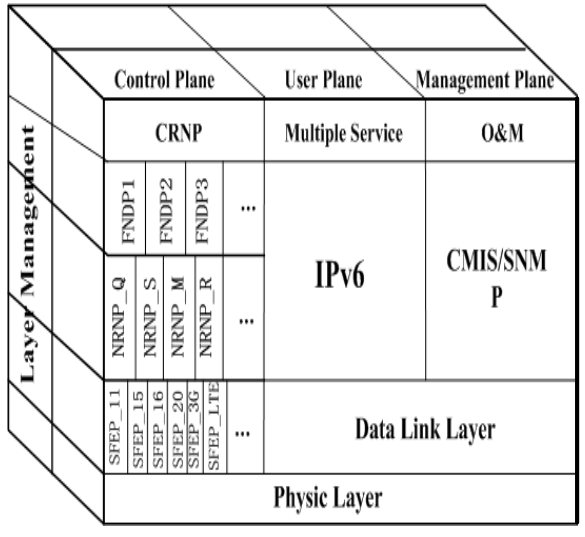

Fig. 4. Trinity Telecom model

structure. Key issue of protocol model framework study as mentioned before, the rapid growth of service and transmission technology of future ubiquitous network present new challenges to network technology. As indicated in figure 3, although the slender hourglass model of IP network protocol takes advantages in the promotion of business innovation and transmission technology integration, it is useless when facing of massive data management. Figure 4 describes a trinity Telecom model, which is for large-scale network operation and management, but due to its closed and rigid structure, it still impossible to completely solve the constraints on innovation, even after introducing the IP protocol. In study of the network architecture, future architecture is no longer stick to cellular network or hotspots network, one transmission technology to one network form, superposition of a variety of network forms will be the main way of network coverage.

Based on above analysis, in the study of network protocol model, getting rid of the simple control of slender model and the closed structure of three-dimensional model, proposing business and transmission based independent control mode is very important. The new mode will no longer strictly follow the protocol rules, but propose a protocol framework for control of special business and transmission. In addition, limited network form applies to particular technology and scenario. In future study, exploring superposition network form according to the wireless environment and application needs, and propose smart network control mechanism is the key solution of the problem.

\section{Key TeChNOlogies fOR Future Wireless NETWORKS}

In this section, we'll discuss some key technologies for future wireless network from three different points of view, the service level, the management level and the transmission level.

Traditional application research emphasizes on system design and implementation. Application technology development has two important characteristics including rapid revolution 


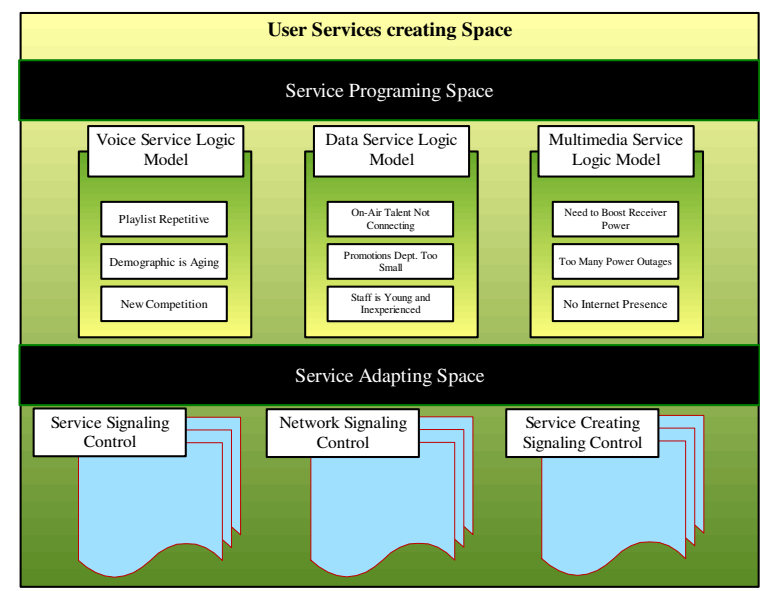

Fig. 5. Cubic model

and close association with society, which make application technology study a kind of social-related science. It is necessary to focus on the above two characteristics with combination of technology developing trend in order to recognize the developing trend of future application system. The first task of application research is to design a service platform which can converge more creative ideas and technologies, i.e. a selfbuild platform as show in figure 5 . This platform consists of modules of users, application logic, and signal control. On top of this platform, end users can create any communication service according to their interest and requirement. What's more, we should study the integration of different information transport modes, automatical selection of unicast, multicast or broadcast services. Then with the every growing demand for the network, in terms of security, we should also study how to provide a reliable secure network and regulate our activity in the control of law and virtue.

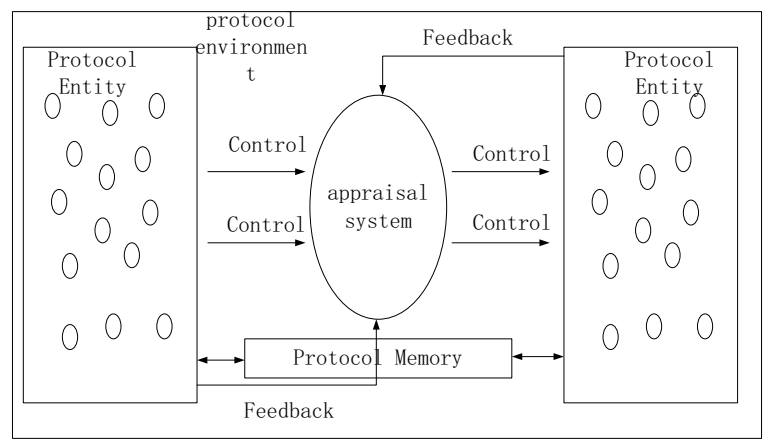

Fig. 6. Cubic model

In the control level research, we need to study the network control technology in post-IP-age, especially the precise network control technology in the link-level, based on the complex service types and different transmission links. Current research on network control covers many aspects, including congestion control when large scale traffic occurs, disturbance control, and virus control. Until present, there is no existing controlling technology to implement intelligent control on network. Therefore, it is necessary to design intelligent network control method for future wireless network. As shown in figure 6, we design an intelligent protocol control model. Some intelligent entities are designed in the model, which has feedback capacity and can assess on controlling results.In real network deployment, we need to consider the intelligence protocol model intensify the network signaling control and establish a complex nervous signaling system. The aim of the research and implementation of signaling system is to prevent the emergence phenomenon caused by paralyticae hidden harm to social securities strengthening the precise control in security affairs.

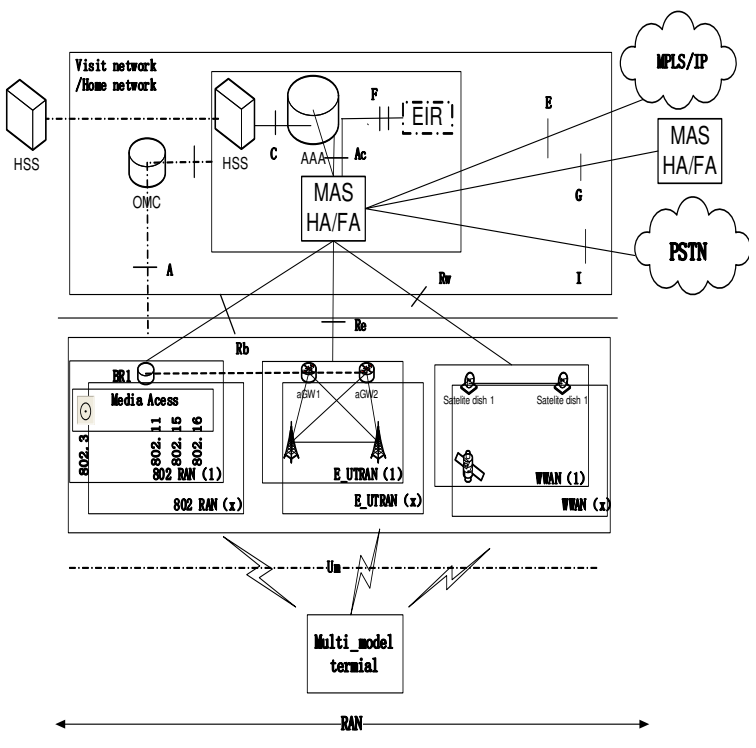

Fig. 7. Cubic model

Heterogeneous mixture is the major characteristic in future wireless network transmission system, that is, fiber-based transmission in the core network, and various access technologies in the wireless access network. In the access network, there're different standards which connect mobile terminals together. For wireless transmission systems, as described a heterogenous network architecture in the 7, there are three major research areas. From the physical transmission point of view, many literatures have focused on studying transmission characteristics of different radio links and adaptive modulation and coding scheme to maximize radio link transmission capacity. The second research area focuses on the intelligent radio resource management technologies. In case of fixed wireless transmission scenario, intelligent media control technology plays a key role in achieving the theoretic radio capacity. The third research area focuses on interference cancellation. When data transmits on different radio links simultaneously, it will cause interference to each other. So we need to study the 
transmission configuration in the access networks to establish a collaborative communication system.

\section{CONCLUSION}

In this paper, we provided a brief survey on the framework and evolution trends for future wireless network from a new perspective. Based on the analysis of some complex network phenomenons, we proposed a novel architecture for future wireless network and presented some hotspots for future research in this area. Finally, some research methods were discussed in-depth from philosophical level, system level and technical level.

\section{REFERENCES}

[1] Amin, "North America's Electricity Infrastructure: Are We Ready for More Perfect Storms?',IEEE Security and Privacy Magazine, Vol. 1, No.5, pp. 19-25, September/October 2003

[2] Xiangning Lin, Zhiqian Bo, " Comparative Analysis of Blackout Data from North America Power Grids Using the Highly Optimized Tolerance Model", 2008 IEEE Power and Energy Society General Meeting

[3] G. Andersson, P. Donalek, R. Farmer, N. Hatziargyriou, "Causes of the 2003 Major Grid Blackouts in North America and Europe, and Recommended Means to Improve System Dynamic Performance",IEEE TRANSACTIONS ON POWER SYSTEMS, VOL. 20, NO. 4, NOVEMBER 2005

[4] Giuseppe Serazzi and Stefano Zanero, "Computer Virus Propagation Models", http://www.elet.polimi.it/upload/zanero/papers/zaneroserazzi-virus.pdf

[5] Pastor-Satorras R., Vespignani A., "Epidemics and immunization in scalefree networks", in Handbook of Graphs and Networks, Bornholdt S., Schuster H.G. (Eds.), WILE-YVCH publisher,2003.

[6] Yamir Moreno, Javier B. Gmez, and Amalio F. Pacheco, "Epidemic incidence in correlated complex networks", Phys.Rev.E.68.035103,2003.

[7] Justin Balthrop, Stephanie Forrest, M. E. J. Newman, Matthew M.Williamson, "Technological Networks and the Spread of Computer Viruses", SCIENCE VOL 30423 APRIL ,2004.

[8] Murthy, E Krishnamurthy,' Can Artificial Life Emerge in a Network of Interacting Agents?", 2006 - en.scientificcommons.org

[9] Greg Goth, "Redefining the Server as Home Networks Emerge," IEEE Internet Computing, vol. 12, no. 2, pp. 7-9, March/April, 2008

[10] Marko H?nnik?inen ,etc,'Trends in personal wireless data communications", Computer Communications Volume 25, Issue 1, 1 January 2002, Pages 84-99

[11] D Kotz, K Essien ,'Analysis of a campus-wide wireless network", Wireless Networks, 2005 - Springer"

[12] JL Kennington, " A survey of linear cost multicommodity network flows" Operations Research, 1978 - jstor.org

[13] CE Jones, KM Sivalingam, P Agrawal, JC Chen ,"A survey of energy efficient network protocols for wireless networks", Wireless Networks, 2001 